\title{
High Frequency Current Sensing using Optical Fiber Micro-wire
}

\author{
G. Y. Chen, M. Belal, Y. Jung, G. Brambilla, and T. P. Newson
}

ORC, University of Southampton, Southampton, SO17 1BJ, UK

Fiber-optic current sensors exploiting the Faraday effect have attracted a great deal of interest due to their wide dynamic range, robustness and remote sensing capability. Recently a new approach to current sensing using optical fiber micro-wire (OFM) with $5 \mu \mathrm{m}$ diameter in the configuration of a micro-coil (MC) has been proposed and successfully demonstrated [1]. It featured high compactness, robustness, configurability and excellent confinement of light and was able to demonstrate its ability to sense currents with a $1 \mu$ s rise time. In this paper we present a major improvement in the current sensing bandwidth, demonstrating a capability to detect a fast current pulse with pulse width (full width at half maximum: FWHM) of $6.7 \mathrm{~ns}$.

A $5 \mu \mathrm{m}$ diameter, $50 \mathrm{~mm}$ long OFM was tapered from a conventional single-mode fiber (Corning SMF-28) by locally heating and stretching using the modified flame brushing technique [2]. The OFM was precisionwrapped around a $0.82 \mathrm{~mm}$ diameter, $40 \mathrm{~mm}$ long copper wire. The length of the OFM coiled around the wire was $\sim 30 \mathrm{~mm}$. The structure is a helical coil of circular cross-section, and has 10 turns with a pitch of $\sim 70 \mu \mathrm{m}$ between each winding to prevent mode coupling leading to unwanted resonance effects. Robustness to the sample was provided by embedding the MC within a layer of polymer (EFIRON UV-373) and cured by ultraviolet (UV) light. An optical loss of $1.1 \mathrm{~dB}$ was recorded at $1550 \mathrm{~nm}$, before and after packaging.

(a)

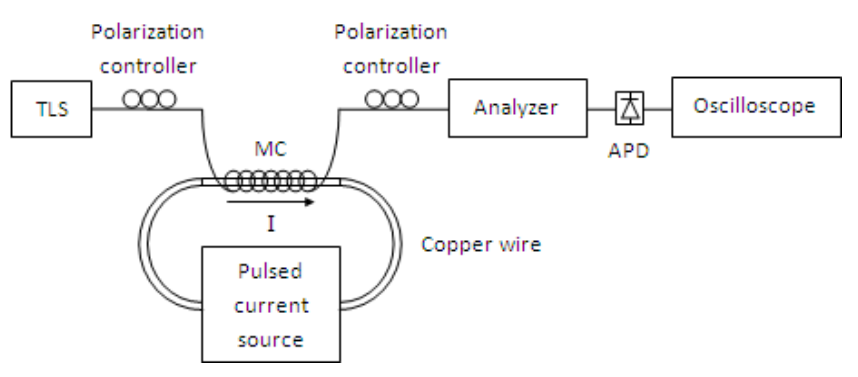

(b)

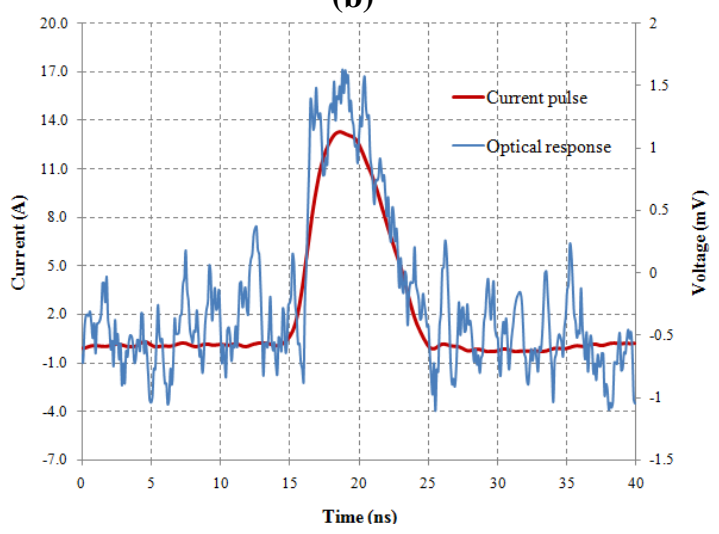

Fig. 1 (a) Layout of optical fiber micro-wire current sensing system and (b) time-domain measurement (no averaging) of optical response superimposed with current pulse.

As shown in Fig. 1(a), linearly-polarized light at $1550 \mathrm{~nm}$ was launched into the MC via a polarization controller, which was used to align the input polarization state along one axis of the MC. A fast pulsed current was generated by a modified pulsed laser diode driver and connected to the copper wire inside the MC. The Faraday effect causes an angular rotation $(\theta)$ of the polarization state of light, given by Eq. (1).

$$
\theta=2 \pi r N V B=\mu_{0} N V I
$$

where $r$ is the radius of the fiber coil, $N$ is the number of turns, $V$ is the Verdet constant of silica, $B$ is the magnetic field, $\mu_{0}$ is the magnetic permeability and $I$ is the current. After exiting the MC, light passes through a second polarization controller which is used to adjust the azimuth of the emerging light $45^{\circ}$ to the axis of the analyzer. The change in polarization rotation translates into a change in intensity. Fig. 1(b) shows the monitored current pulse (FWHM of $6.7 \mathrm{~ns}$, rise time of $4.4 \mathrm{~ns}$ and fall time of $6.8 \mathrm{~ns}$ ) and the corresponding optical response. It is evident that the bandwidth of the optical current sensor matches or even exceeds the bandwidth of the monitored electrical pulse.

In conclusion, we have experimentally demonstrated a MC current sensor with a sufficiently short response time to identify a fast current pulse with temporal transitions of the order of a few ns. The theoretical response time governed by the MC length is 150 ps corresponding to $2.3 \mathrm{GHz}$ bandwidth, assuming a pulse duration bandwidth product of $\sim 0.35$. There is a trade-off between responsivity and response time of the current sensor. A smaller number of turns will result in a weaker Faraday effect, but with the benefit of faster response time.

\section{References}

[1] M. Belal, Z. Song, Y. Jung, G. Brambilla, and T. P. Newson, “Optical fiber microwire current sensor”, Opt. Lett. 35, 3045 (2010).

[2] G. Brambilla, "Optical fibre nanowires and microwires: a review", J. Opt. 12, 9 (2010). 